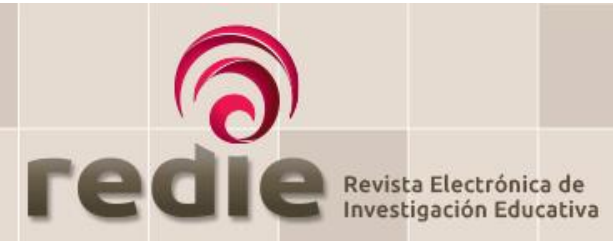

\title{
Desarrollo de un instrumento para evaluar actitudes hacia la formación universitaria: un estudio de caso en la UAM-I
}

\section{Attitude Questionnaire about University Education: a Case Study at UAM-I}

\author{
Graciela Ontiveros Ruiz (1) goir31@gmail.com \\ Martha Diana Bosco Hernández (2) bosco@unam.mx \\ Consuelo Díaz (1) ditc@xanum.uam.mx \\ Raquel Valdes Cristerna (1) ravc@xanum.uam.mx \\ Rubicelia Vargas Fosada (1) ruvf@xanum.uam.mx \\ Margarita Viniegra Ramirez (1) mvr@xanum.uam.mx
}

(1) Universidad Autónoma Metropolitana-Iztapalapa

(2) Universidad Nacional Autónoma de México

(Recibido: 29 de mayo de 2017; Aceptado para su publicación: 18 de diciembre de 2017)

Cómo citar: Ontiveros, G., Bosco, M. D., Díaz, C., Valdes, R., Vargas, R. y Viniegra, M. (2019). Desarrollo de un instrumento para evaluar actitudes hacia la formación universitaria: un estudio de caso en la UAM-I. Revista Electrónica de Investigación Educativa, 21, e38, 1-13. doi:10.24320/redie.2019.21.e38.1998

\section{Resumen}

Considerando que las actitudes tienen una gran incidencia en el desempeño académico de los alumnos, se reporta la elaboración y validación de un instrumento para evaluar las actitudes hacia la formación universitaria de estudiantes en Ciencias Básicas e Ingeniería. El instrumento fue validado por expertos y el análisis de consistencia interna reportó un Alfa de Cronbach total de 0.96. Se aplicó a un grupo de 123 estudiantes; el análisis factorial (Bartlett de 131.38 con $p<0.0001$ y kMO de 0.842) permitió identificar dos factores asociados a procesos grupales e individuales. El análisis de conglomerados de cada factor (Anova $p<0.001$ ) permitió la identificación de tres niveles en la escala de actitudes. Se construyó la descripción de los perfiles de actitudes hacia la formación universitaria para cada nivel en cada dimensión. Se ejemplifica la descripción del perfil actitudinal en dos casos de alumnos en formación propedéutica.

Palabras clave: Actitud del estudiante, educación superior, ciencias, Instrumento de medición, evaluación del estudiante.

\begin{abstract}
Considering that the attitudes have a great importance in the academic performance of the students, the elaboration and validation of an instrument to evaluate the attitudes towards university formation of students in Basic Sciences and Engineering is reported. The instrument was validated by experts and the internal consistency analysis reported a total Cronbach Alpha of 0.96. It was applied to a group of 123 students; factor analysis (Bartlett of 131.38 with $p<0.0001$ and KMO of 0.842 ) allowed to identify two factors associated with group and with individual processes. The cluster analysis of each factor (Anova $p<$ 0.001) permitted the identification of three levels in the attitudes scale. The description of the attitudes profiles towards university formation was constructed for each level in every dimension. The description of the attitudinal profile is exemplified for two students in propaedeutic training.
\end{abstract}




\section{Introducción}

Desde hace más de diez años, la División de Ciencias Básicas e Ingeniería (CBI) de la Universidad Autónoma Metropolitana Unidad Iztapalapa (UAM-I) ofrece a los alumnos de nuevo ingreso un programa propedéutico denominado Cursos Complementarios (cc), con la finalidad de prepararlos en conocimientos y habilidades matemáticas, de comunicación científica y de resolución de problemas, requeridos (UAM-I, CBI, 2008) para iniciar sus estudios en cualquier licenciatura de esa División ${ }^{1}$, además de brindarles un espacio de inducción a la vida universitaria.

En los cc los alumnos presentan una evaluación diagnóstica denominada Examen General de Cursos Complementarios (EGCC) que, además de servir para conocer el nivel de conocimientos y habilidades que tienen los jóvenes al ingresar a CBI, también ha sido una referencia para evaluar el aprendizaje logrado a través del mismo programa, ya que se aplica antes de iniciarlo y al concluirlo. Los resultados de la primera aplicación son uno de los criterios que utiliza la División de CBI para decidir si un alumno debe inscribirse a este programa propedéutico o puede cursar directamente las asignaturas del primer trimestre de su licenciatura.

Las evaluaciones hechas mediante el EGcc muestran que con los cc los alumnos incrementan en general sus conocimientos y habilidades matemáticas y en comunicación en las Ciencias e Ingeniería, como lo reportan los informes anuales de la coordinación de los mismos cursos; y por lo que se refiere a su trayectoria académica también se observa en informes de seguimiento que los alumnos que han cursado cc tienen mayor permanencia en sus estudios que los alumnos que ingresan directamente al trimestre uno (UAM-I, CBI, 2010-2013).

Además de la utilidad del EGCC para conocer el nivel de conocimientos con que los alumnos ingresan a la División de CBI y para evaluar los CC, se considera importante conocer las actitudes de los alumnos hacia sus estudios. Éstas han surgido como tema recurrente entre los profesores que han impartido el curso, ya que son un aspecto importante que puede favorecer o complicar el desarrollo académico de los estudiantes.

Por lo anterior, se decidió elaborar un instrumento que permitiera evaluar sistemáticamente las actitudes hacia la formación universitaria que tienen los alumnos de nuevo ingreso. Se entiende la formación universitaria como el conjunto de procesos sociales de preparación y conformación del sujeto, referidos a fines precisos para un posterior desempeño laboral (Bosco, 2009, p. 323). Contar con un instrumento válido y confiable para nuestra población permitirá recabar información sobre este tipo de comportamiento que, aunque se contempla en los perfiles de ingreso y de egreso de CBI, no se ha investigado formalmente.

El grupo de trabajo que formuló el presente instrumento está integrado por académicas de las disciplinas de Ingeniería Biomédica, Matemáticas, Pedagogía, Psicología y Química, cuyo objetivo fue desarrollar un instrumento, su validación, aplicación y resultados para dar inicio a las investigaciones sobre las actitudes de los alumnos de la División de CBI de la UAM-I.

De acuerdo con Fishbein y Azjen (1975), se considera la actitud como una predisposición relativamente estable a evaluar en un determinado objeto social (persona, grupo, suceso o situación). La evaluación a la que nos referimos puede ser favorable, desfavorable o inclusive ambivalente. Se entiende que la predisposición implica una postura subjetiva respecto al objeto, por lo que la actitud no se puede conocer directamente, sino por la manera en que el individuo se expresa, actúa, se comporta e interactúa con los demás. Por ejemplo, la expresión de un estudiante "prefiero trabajar solo que con otros compañeros" se puede tratar como un indicador de una actitud desfavorable hacia el trabajo colaborativo; pero si en vez de comentar esto, el estudiante manifestara su gusto por intercambiar puntos de vista con sus compañeros, entonces sería un indicador que apunta a una actitud favorable hacia el mismo objeto. En este punto, Ajzen (2005) plantea que la actitud es una variable latente, que ha de ser inferida de ciertas

\footnotetext{
${ }^{1}$ Oferta académica División de cBl: www.cbiuami.org/index.php/oferta-academica/licenciaturas
} 
respuestas mensurables y que refleja, en una última instancia, una evaluación global positiva o negativa del objeto en cuestión.

Las actitudes se forman con base en los conocimientos y creencias adquiridas mediante la interacción con los demás, y en experiencias vividas directamente, procesos en los que está implicado el objeto de la actitud. Así, una actitud puede tener, aunque en intensidades distintas, tres tipos de componentes: cognitivo, afectivo y conductual. ${ }^{2}$

El componente cognitivo es el conocimiento o creencia que la persona tiene sobre un objeto social. De acuerdo con Rodrigues (1976), para que se genere una actitud en relación con un objeto determinado es necesario que exista alguna representación cognoscitiva de dicho objeto. Dicha representación despierta a su vez una carga afectiva o una emoción hacia el mismo objeto, que es el componente afectivo. Es esta emoción la que finalmente define la actitud favorable o desfavorable. Con relación a este componente, Fishbein y Ajzen (1975) lo señalan como el más característico y distintivo de las actitudes.

El componente conductual se refiere a las tendencias, disposiciones e intenciones hacia el objeto, así como las acciones dirigidas al mismo (Morales, 1994). De acuerdo con este autor, los tres componentes coinciden en que son evaluaciones del objeto de la actitud. Así, la información que se tiene del objeto puede ser favorable o desfavorable, las emociones pueden ser positivas o negativas, y la conducta e intenciones de conducta pueden ser de apoyo o desaprobación.

A continuación, se enlistan las características de las actitudes consideradas relevantes para este estudio. Las actitudes son:

- Una tendencia relativamente duradera para evaluar de cierto modo un objeto social (Baron y Byrne, 2005). ${ }^{3}$

- Una evaluación del objeto social que puede ser a favor o en contra, o ambivalente.

- Experiencias con respecto a un objeto social: persona, grupo, objeto o situación.

- Experiencias subjetivas, es decir, son procesos que experimenta la persona en su conciencia.

- Evaluaciones subjetivas que se conocen sólo a través de su expresión, en lenguaje verbal o no verbal.

- Variables latentes, consideradas como una "semilla" que bajo ciertas condiciones suele "germinar en comportamiento" (Hernández, Fernández-Collado y Baptista, 2006).

- Una predisposición o grado de compromiso a la acción.

- Una predisposición que se aprende.

Vale la pena resaltar las tres últimas características en el ámbito universitario, por la posibilidad de promover en los estudiantes actitudes congruentes con su formación profesional.

Debido a que las actitudes son consideradas elementos clave en la adquisición de nuevos conocimientos y habilidades personales y sociales, también se tomó en cuenta la Teoría de la Acción Razonada de Fishbein y Azjen (2010), ya que explica cómo se vinculan éstas con la conducta o el comportamiento e identifica los elementos que participan en todo proceso de toma de decisión. Así, esta teoría plantea que la decisión de implicarse en una conducta particular es el resultado de un proceso racional, en el cual la persona considera las opciones que tiene respecto a la conducta, y evalúa sus consecuencias o resultados de cada una para tomar una decisión y llevar a cabo un plan de acción. Esta decisión se refleja entonces en las intenciones conductuales, las cuales influyen fuertemente en la conducta. Las intenciones están, a su vez, determinadas por información o creencias que el sujeto tiene respecto a una conducta particular. Se

\footnotetext{
${ }^{2}$ Estos componentes es una clasificación clásica del comportamiento humano, y es utilizada con frecuencia en la investigación de actitudes. Ésta fue útil en este estudio como guía en la elaboración de los reactivos del instrumento desarrollado.

${ }^{3}$ Para Baron y Byrne, la fuerza de una actitud depende del grado de intensidad, importancia, conocimiento y accesibilidad que el objeto en cuestión represente para la persona. Por intensidad, se refieren a la reacción emocional provocada por el objeto de la actitud; por importancia, al interés implicado en la actitud; por conocimiento, cuánto se sabe acerca del objeto de la actitud; y por accesibilidad, a la facilidad con que se recuerda la actitud.
} 
distinguen tres tipos de información o creencias: El primero se refiere a las creencias que tiene el mismo sujeto respecto a una determinada conducta, en la que está implicado un afecto favorable o desfavorable hacia la misma conducta (actitud); el segundo tipo son las creencias que tiene el sujeto de las personas que le son importantes respecto de lo que piensan de la conducta en cuestión, llamadas normas subjetivas; y el tercer tipo de creencias se refiere al control conductual percibido. Éste es la valoración de las propias habilidades para realizar la conducta, así como de las facilidades o limitaciones medioambientales percibidas para la realización de la misma conducta. Así, las intenciones son el resultado del proceso subjetivo de la combinación de las creencias ya mencionadas, aunque es de esperarse que, por el cambiante acontecer de la vida de las personas, la importancia o peso de estas creencias varíen de un comportamiento a otro y de un individuo a otro.

A manera de resumen, y de acuerdo con Morales (1994), el concepto central de esta teoría es la intención conductual, ya que es la causa inmediata de la conducta, y se puede definir como el juicio probabilístico que emite una persona acerca de ejecutar o no un comportamiento. La intención conductual se forma básicamente por dos factores, uno de tipo personal (que son las actitudes hacia determinada conducta) y otro social (que son las normas subjetivas). De acuerdo con Rizo (2005):

El énfasis no se encuentra ni en el sistema social ni en las relaciones funcionales que se dan en la vida en sociedad, sino en la interpretación de los significados del mundo y las acciones e interacciones de los sujetos sociales. Del mundo conocido y de las experiencias intersubjetivas compartidas por los sujetos en todas las dimensiones de la vida social. (p. 1).

Lo que redundará en un beneficio colectivo de la vida universitaria.

\section{Método}

Con base en la consulta de estudios previos sobre actitudes, similares al presente (Bas, Kubiato y Murat, 2016; García, 2013; García y Hirsch, 2006; Gargallo, Pérez, Fernández y Jiménez, 2007; Marín y Alarcón, 2013; Peralta, Ramírez y Castaño, 2006; Pliego, Contini, Odetti y Tiburzi, 2004; Sáenz, Sánchez-Pérez, Luenga y Jarillo, 2007; Senay, 2013; Topala, 2014), en la teoría de actitudes propuesta por Fishbein y Ajzen (2010), la técnica Likert para la construcción de escalas y los indicadores de las actitudes favorables, se diseñó un instrumento que permitiera valorar las actitudes de los alumnos hacia la formación universitaria y se construyeron perfiles actitudinales para un grupo de estudiantes de Ciencias Básicas e Ingeniería de la UAM-I.

Se llevó a cabo una investigación exploratoria porque no existen estudios previos en la División de CBI sobre actitudes de los alumnos hacia la formación universitaria. Asimismo, se realizó un estudio descriptivo que da cuenta de las dimensiones y componentes de cada uno de los reactivos del instrumento y se elaboró un estudio correlacional para conocer el grado de relación que existe entre los reactivos propuestos para la validación del instrumento mediante el análisis de consistencia interna.

La metodología se describe en tres fases: La primera da cuenta de la identificación de las dimensiones a valorar, elaboración de sus indicadores, y elaboración de los reactivos del cuestionario; la segunda se refiere a la validación del instrumento a través del juicio de expertos, la prueba piloto, y el análisis de consistencia interna y la tercera consiste en la aplicación del cuestionario ya validado, el correspondiente análisis estadístico y la definición de los perfiles.

\subsection{Fase 1. Diseño}

Para la elaboración del cuestionario se tomaron en cuenta las actitudes descritas en el Sistema Divisional a nivel Licenciatura CBI (UAM-I, CBI, 2008) y el programa de CC (UAM-I, CBI, 2013), tales como: disposición para el trabajo colaborativo en diferentes escenarios, disciplina de trabajo individual y de grupo, capacidad para interactuar con sus compañeros y profesores bajo un espíritu de respeto, equidad y solidaridad, responsabilidad de su propio aprendizaje, desarrollo de hábitos y técnicas de estudio, reconocimiento de la comunicación oral y escrita en la apropiación del conocimiento, organización y planificación del trabajo 
académico, tolerancia a la frustración, capacidad de análisis y síntesis, creatividad y desarrollo del pensamiento crítico, e integración de conocimientos para resolución de problemas situados.

Analizado el perfil anterior se procedió a establecer las dimensiones a evaluar, así como sus indicadores favorables, a fin de guiar la elaboración de los reactivos del cuestionario. En la tabla I se muestra la relación entre los componentes del perfil y las dimensiones a medir. Enseguida se presentan los indicadores.

Tabla I. Componentes del perfil y dimensiones a medir

\begin{tabular}{l|l}
\hline Componentes del perfil & Dimensiones \\
\hline Disposición para el trabajo colaborativo & $\mathrm{TE}, \mathrm{RI}$ \\
Capacidad para convivir en un medio universitario & $\mathrm{RI}$ \\
Disposición y capacidad para el estudio independiente & $\mathrm{HT}$ \\
Capacidad para organizar y planificar su trabajo académico & $\mathrm{HT}, \mathrm{MO}$ \\
Habilidades de comunicación oral y escrita & $\mathrm{CO}$ \\
Motivación hacia su formación profesional & $\mathrm{MO}$ \\
Capacidad de análisis y síntesis, y pensamiento crítico & $\mathrm{PC}, \mathrm{RP}$ \\
\hline Habilidad en el uso de técnicas para la resolución de problemas & $\mathrm{RP}$ \\
\hline
\end{tabular}

Nota: $\mathrm{TE}$ = Trabajo colaborativo y en equipo, RI = Relaciones interpersonales, $\mathrm{HT}$ = Hábitos y técnicas de estudio, $\mathrm{CO}=$ Comunicación oral y escrita, $\mathrm{MO}=$ Motivación personal, $\mathrm{PC}=$ Pensamiento crítico, RP = Resolución de problemas.

\section{Indicadores para cada dimensión}

Trabajo colaborativo y en equipo (TE)

- Identifica la diferencia entre grupo y equipo.

- Sabe que todo grupo puede llegar a ser un equipo mediante el desarrollo de sus miembros.

- Reconoce las dificultades más frecuentes en el TE, así como sus ventajas en cuanto aprendizaje y trabajo logrados.

- Tiene la misma disposición para el TE como para el trabajo individual.

- Está dispuesto a superar las dificultades que se presenten en el TE.

- Considera que puede aprender de sus compañeros.

- Reconoce la importancia de las interacciones enfocadas a la tarea del grupo y las enfocadas a la constitución y mantenimiento del mismo, para un buen desarrollo del TE.

- Gusta de compartir sus conocimientos.

- Reconoce a los demás sus aciertos y ante los demás, sus desaciertos.

- Reconoce la importancia de aplicar el proceso administrativo en el TE.

- Identifica las características de un equipo de alto rendimiento.

Relaciones interpersonales (RI)

- Reconoce la importancia de aplicar los valores de respeto, tolerancia, equidad, justicia y solidaridad, para una convivencia armónica y constructiva en la comunidad universitaria.

- Considera que es posible el acuerdo entre las personas, aunque éstas difieran en ideas, gustos o maneras de percibir.

- Muestra interés en conocer a sus compañeros.

- Tiene disposición a establecer una comunicación sincera con sus compañeros y profesores.

- Muestra empatía en sus relaciones interpersonales.

- Evita los prejuicios en su trato con los demás.

- Está dispuesto a ayudar a los demás.

- Muestra interés en conocer sus derechos y obligaciones como estudiante.

- Defiende sus derechos cuando considera que no se toman en cuenta. 
- Sabe diferenciar entre los deberes de estudiante y las muestras de amistad.

Hábitos y técnicas de estudio (HT)

- Tiene preparado un lugar específico para estudiar.

- Organiza sus diversas actividades de manera racional.

- $\quad$ Le gusta ser puntual.

- Estudia con un plan previo.

- Acostumbra anotar sus dudas para investigarlas o preguntarlas a los profesores.

- Toma notas en sus clases.

- Respeta el tiempo asignado para estudiar.

- Se asegura de entender las tareas a realizar.

- Cumple con sus tareas en tiempo.

- Reconoce la importancia de la práctica para el dominio de una habilidad.

- Elige las horas más adecuadas para estudiar.

- Lee con cuidado las instrucciones de los materiales didácticos.

- Consigue el material que necesitará para sus cursos.

- Estudia otros materiales además de los indicados por los profesores.

Comunicación oral y escrita (co)

- Tiene inclinación por la lectura.

- Acostumbra subrayar las partes clave en sus lecturas.

- Muestra interés por aprender el lenguaje científico.

- Identifica las secciones que deben contener un documento de acuerdo a su contexto.

- Revisa la redacción y ortografía de sus trabajos antes de entregarlos.

- Elabora sus trabajos escritos con base en sus propias ideas, es decir, no recurre al "copiar y pegar".

- Se propone para exponer cuando se necesita.

- Tiene gusto por participar en debates.

- Sabe escuchar.

- Le gusta la comunicación directa y clara.

Motivación personal (MO)

- Tiene interés por conocerse a sí mismo.

- Considera al conocimiento y superación personal entre sus valores más preciados.

- Le gusta el ambiente universitario.

- Se conduce en la vida con propósitos claros.

- Confía que con su dedicación logrará sus metas.

- Es perseverante.

- Procura su salud física, mental y emocional.

- Disfruta lo que hace.

Pensamiento crítico (PC)

- Trata de informarse de todo lo que le involucra.

- Busca participar en discusiones intelectuales.

- Analiza la información disponible de un tema determinado antes de opinar o tomar una decisión.

- Apoya su aprendizaje más en la razón que en la memoria.

- Evalúa la información recibida por su claridad y relevancia.

- Profundiza en el conocimiento, formulando diferentes preguntas. 
Resolución de problemas (RP)

- Sabe que entender el problema es primordial para su resolución.

- Se apoya más en los conocimientos que en la intuición para resolver los problemas.

- Analiza diferentes estrategias para resolver un problema.

- Evalúa resultados.

- Enfrenta los problemas que le atañen.

Para la construcción del instrumento se elaboró un conjunto de 147 reactivos (en promedio 21 por cada dimensión), se consideró una escala Likert de cinco opciones. Cada reactivo representó un enunciado favorable-desfavorable sobre diversos aspectos o dimensiones de estudio de la actitud hacia la formación universitaria. En los enunciados favorables cada opción se ponderó de la siguiente manera: totalmente de acuerdo (4), de acuerdo (3), indiferente (2), en desacuerdo (1), totalmente en desacuerdo (0), y en los enunciados desfavorables la ponderación varía en sentido inverso.

La puntuación total de cada encuestado se obtuvo mediante la suma de las puntuaciones de cada uno de los reactivos y lo ubicó en un punto de la escala de las variables medidas.

Para completar el banco de reactivos se tomaron cinco enunciados del instrumento Inventario de Técnicas y Actitudes de Estudio ${ }^{4}$, por considerarlos pertinentes para cubrir las actitudes a evaluar en las dimensiones de Comunicación, Motivación y Pensamiento Crítico.

Se verificó que en cada dimensión hubiera reactivos con referencia a los tres componentes de las actitudes. El cuestionario está balanceado en cuanto al número de reactivos favorables y desfavorables dentro de cada dimensión a estudiar, se procuró así la neutralidad en la escala.

Para aplicar el cuestionario se utilizó Lime Survey ${ }^{5}$, una aplicación para gestionar encuestas vía Internet. Esta aplicación permitió que el encuestado pudiera agregar un comentario en cada reactivo que le parecía confuso o poco claro, y en esos casos también indicara la causa de tal confusión.

\subsection{Fase 2. Validación}

Para el proceso de validación se envió el instrumento a tres jueces expertos académicos de la Facultad de Psicología de la Universidad Nacional Autónoma de México y se aplicaron las correcciones sugeridas por ellos.

La prueba piloto del instrumento se aplicó al total de alumnos que participaron en los Cc (180 de nuevo ingreso), de todas las licenciaturas que se imparten en la División de CBI.

El cuestionario se presentó a los alumnos con los reactivos en orden aleatorio, mezclando reactivos de diferentes dimensiones para evitar posibles patrones en el orden de las preguntas. Además, se aplicaron dos versiones del cuestionario para controlar los posibles sesgos derivados de las circunstancias de la aplicación, variando entre ambos el orden de presentación de los reactivos.

Por último, se aplicó el análisis de consistencia interna empleando el paquete estadístico NCSS 8. Los resultados de la validación se presentan en la sección correspondiente.

\subsection{Fase 3. Construcción de perfiles}

Una vez validado el cuestionario se aplicó a un grupo de 123 alumnos voluntarios de todas las

\footnotetext{
${ }^{4}$ Instrumento desarrollado en 1992 por Rosado, Aduna y García, en la UAM-I, Sección de Orientación y Selección, basado en la Guía de Estudio Efectivo de W. F. Brown.

${ }^{5}$ Lime Survey es un software libre que administra encuestas (www.limesurvey.org)
} 
licenciaturas de CBI que participaron en los CC.

Con estos datos se llevó a cabo un análisis factorial exploratorio con los totales de cada dimensión usando los métodos de componentes principales y rotación varimax. Para evaluar el modelo factorial se realizó la prueba de esfericidad de Bartlett y se calculó el índice KMO.

Se aplicó un análisis de conglomerados (clusters) a fin de identificar diferentes grupos de alumnos con una actitud hacia la formación universitaria similar dentro de cada conglomerado. En este análisis se utilizó la distancia euclidiana como medida de distancia entre los elementos. Para la formación de conglomerados se aplicó el método jerárquico de Ward. Se aplicó Anova en la confirmación de diferencias entre clusters. En todos estos análisis se utilizó el paquete sPSS Statistics 17.

\section{Resultados}

Como primer resultado de la validación del instrumento se atendieron los comentarios de los alumnos sobre la falta de claridad de los reactivos y se descartaron los que fueron señalados como confusos. De igual forma, se consideraron las observaciones de los expertos consultados.

Con los datos obtenidos de los 180 alumnos de la prueba piloto se llevó a cabo el análisis de consistencia interna dentro de cada dimensión. Como resultado de este análisis se descartaron un total de 52 reactivos y se obtuvo un Alfa de Cronbach de 0.9660 para todo el instrumento. En la tabla II se presenta el Alfa de Cronbach alcanzado en cada dimensión y en la prueba total, así como el número de reactivos que se mantuvieron, por dimensión y de manera total. Con estas modificaciones el instrumento se consideró validado con 95 reactivos y listo para aplicarse.

Tabla II. Alfa de Cronbach y número de reactivos, por cada dimensión

\begin{tabular}{l|l}
\hline Dimensión & $\begin{array}{c}\text { Alfa } \\
\text { de Cronbach }\end{array}$ \\
\hline Comunicación (12) & 0.6673 \\
Hábitos y Técnicas (19) & 0.7978 \\
Motivación (15) & 0.7935 \\
Pensamiento Crítico (11) & 0.6195 \\
Relaciones Interpersonales (12) & 0.6036 \\
Resolución de Problemas (16) & 0.7367 \\
Trabajo en Equipo (10) & 0.8224 \\
Total (95) & 0.9660 \\
\hline
\end{tabular}

En cuanto a la construcción de perfiles, con los datos de los 123 alumnos se realizó un análisis descriptivo y se encontró que en 32 reactivos más del $90 \%$ de las respuestas son favorables. Esto implica que son reactivos con baja discriminación y no ayudan a diferenciar a los alumnos.

De la evaluación del modelo factorial se obtuvo un valor del estadístico de la prueba de Bartlett de 131.38 con un valor $p<0.0001$ y un índice KMO de 0.842, por lo que es apropiado aplicar el análisis factorial a estos datos. De este análisis resultaron dos factores, los cuales corresponden al número de valores propios mayores a 1, con los que se obtiene un porcentaje de variación explicado de $73.20 \%$. En el primer factor se ubican las dimensiones MO, HT, PC Y RP y en el otro, la dimensión TE. Las dimensiones RI y CO aparecen en ambos factores. Cabe mencionar que la diferencia encontrada entre estos dos factores consiste en que las dimensiones del primero se asocian con procesos individuales, mientras que la dimensión del segundo se asocia con procesos grupales. También esto explica que las dimensiones RI y co aparezcan en ambos factores, ya que éstas están asociadas tanto con procesos individuales como grupales.

A fin de identificar los diferentes grupos de alumnos de acuerdo con su actitud y definir el perfil de cada grupo se aplicó un análisis de conglomerados por factores: procesos individuales y procesos grupales. Рara realizar este análisis, los reactivos de las dimensiones que resultaron en ambos factores se 
clasificaron en procesos grupales o individuales, distinguiendo en cada reactivo si el planteamiento implicaba o no una relación con más personas. Se identificaron claramente sólo tres conglomerados, con los que se definieron tres niveles de escala para elaborar los perfiles correspondientes. Para confirmar la diferencia entre los tres grupos se aplicó el Anova obteniéndose en todos los casos un valor $p<0.001$.

Para el factor Procesos individuales, en el Grupo I se aglutinaron el $29.3 \%$ de los alumnos, $48.8 \%$ en el Grupo II y 21.9\% en el Grupo III. Para el factor Procesos grupales, el Grupo I abarcó a 8.9\% de los alumnos, $52.9 \%$ en el Grupo II y $38.2 \%$ en el Grupo III.

Cabe resaltar que las respuestas mantienen una tendencia a distribuirse hacia la actitud positiva, aun para el grupo de alumnos que en el análisis de conglomerados quedó ubicado como el que tiene la actitud menos favorable.

En las tablas III y IV se muestran los rangos de puntuación, calculados como porcentaje, de los grupos resultantes. En la tabla III para procesos individuales y en la IV para procesos grupales.

Tabla III. Rangos de puntuación para los tres conglomerados de Procesos Individuales

\begin{tabular}{l|c|c|c}
\hline \multicolumn{1}{c|}{ Dimensión } & $\begin{array}{c}\text { Grupo I: } \\
\text { Actitud poco favorable }\end{array}$ & $\begin{array}{c}\text { Grupo II: } \\
\text { Actitud favorable }\end{array}$ & $\begin{array}{c}\text { Grupo III: } \\
\text { Actitud muy favorable }\end{array}$ \\
\hline Comunicación & $\leq 74.3$ & $74.4-81.9$ & $\geq 82.0$ \\
Hábitos y técnicas de estudio & $\leq 64.3$ & $64.4-75.3$ & $\geq 75.4$ \\
Motivación & $\leq 81.6$ & $81.7-89.6$ & $\geq 89.7$ \\
Pensamiento crítico & $\leq 70.8$ & $70.9-81.4$ & $\geq 81.5$ \\
Relaciones interpersonales & $\leq 79.9$ & $75.0-84.7$ & $\geq 84.8$ \\
Resolución de Problemas & $\leq 72.7$ & $72.8-81.5$ & $\geq 81.6$ \\
\hline
\end{tabular}

Con base en los rangos de puntuación de los tres conglomerados, los tópicos de los reactivos y los indicadores definidos de las actitudes favorables se elaboraron los perfiles de cada grupo, los que permiten describir la actitud de los alumnos hacia la formación universitaria.

Tabla IV. Rangos de puntuación para los tres conglomerados de Procesos Grupales

\begin{tabular}{l|c|c|c}
\hline \multicolumn{1}{c|}{ Dimensión } & $\begin{array}{c}\text { Grupo I: } \\
\text { Actitud poco favorable }\end{array}$ & $\begin{array}{c}\text { Grupo II: } \\
\text { Actitud favorable }\end{array}$ & $\begin{array}{c}\text { Grupo III: } \\
\text { Actitud muy favorable }\end{array}$ \\
\hline Comunicación & $\leq 62.8$ & $62.9-76.0$ & $\geq 76.1$ \\
\hline Relaciones interpersonales & $\leq 63.8$ & $63.9-75.3$ & $\geq 75.4$ \\
\hline Trabajo en equipo & $\leq 54.5$ & $54.6-74.2$ & $\geq 74.3$ \\
\hline
\end{tabular}

Como ejemplo de la interpretación de los resultados de este perfil, en las figuras 1 y 2 se muestran las gráficas que representan los perfiles de un par de alumnos elegidos al azar. 


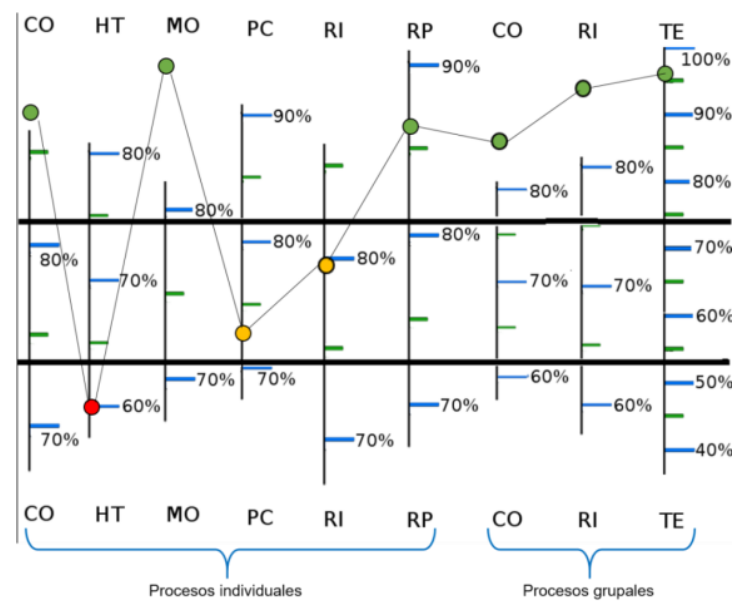

Figura 1. Ejemplo de perfil de una alumna universitaria en formación propedéutica. Rojo en Grupo I (actitud poco favorable), amarillo en Grupo II (actitud favorable), verde en Grupo III (actitud muy favorable)

La gráfica ubica un punto en la escala para cada dimensión. Las líneas gruesas horizontales dividen a los grupos que resultaron del análisis de conglomerados. De acuerdo a la figura 1, la dimensión Comunicación (co) muestra que la alumna tiene un marcado interés por la lectura, por aprender a elaborar trabajos escritos y a exponer en público; tiene habilidad para dirigir su atención y captar las ideas o información importantes de lo que lee y escucha, y cierta facilidad para redactar y exponer. En la dimensión Hábitos y técnicas de estudio (HT) muestra baja inclinación hacia el estudio; mantiene una dependencia hacia sus profesores para su aprendizaje; estudia sólo bajo presión externa; no ve la necesidad de mejorar su manera de estudiar. En Motivación (MO) se observa que tiene metas académicas bien definidas y alta disposición y confianza en poder alcanzarlas no obstante las dificultades que se le presenten; valora en gran medida el conocimiento y la superación personal y cuenta con una autoestima alta. Respecto a Pensamiento crítico (PC) se nota que se informa de los temas que le involucran; tiende a opinar o tomar una decisión de algún asunto sin hacer un análisis y evaluación de la información disponible; su aprendizaje lo apoya más en la razón que en la memoria.

En lo que se refiere a Relaciones interpersonales (RI) le interesa mantener una relación armónica y constructiva con los demás; reconoce poco la importancia que tiene el respeto, la tolerancia y la solidaridad para ello; tiene cierto interés en conocer a sus compañeros, así como en conocer sobre sus derechos y obligaciones como estudiante; se le dificulta defender sus derechos cuando considera que han sido vulnerados y a veces relaja su honestidad por complacer a sus compañeros. En cuanto a Resolución de problemas (RP) se muestra siempre con disposición a resolver problemas, aunque éstos se vean complicados; para resolverlos se asegura de entenderlos bien, selecciona y aplica la estrategia que considera adecuada, se apoya más en sus conocimientos que en su intuición y evalúa resultados. Todas estas dimensiones corresponden a los procesos actitudinales individuales. Respecto a los procesos grupales se observa en co que tiene una alta disposición para comunicarse con sus compañeros; siempre procura comunicarse de manera directa y clara; sabe escuchar y ser empática. Respecto a RI resulta una persona muy sociable y solidaria, dispuesta siempre a ayudar a los demás, dispuesta a integrarse a los grupos cuyos objetivos comparte y a respetar las normas establecidas en ellos; pide ayuda fácilmente cuando la necesita. En Trabajo en equipo (TE) se observa que cuenta con alta disposición, compromiso, interés y disciplina para las tareas grupales; participa con más de las tareas asignadas y mantiene adecuada retroalimentación con sus compañeros de equipo. 


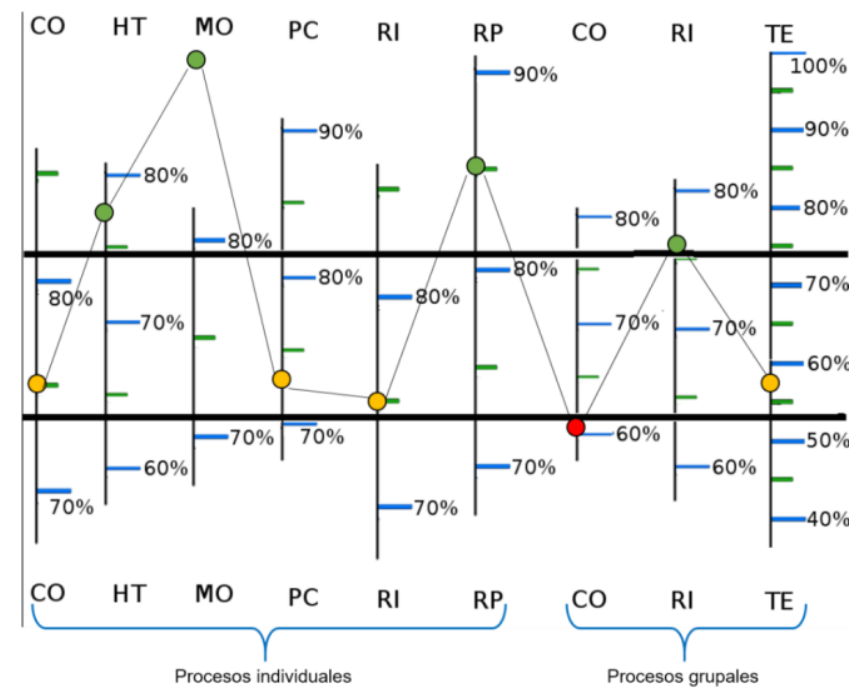

Figura 2. Ejemplo de perfil de un alumno universitario en formación propedéutica. Rojo en grupo I (actitud poco favorable), amarillo en grupo II (actitud favorable), verde en grupo III (actitud muy favorable)

La dimensión co de la figura 2 señala que este alumno muestra mediano interés por la lectura, por aprender a elaborar trabajos escritos y a exponer en público; tiene habilidad para dirigir su atención y captar las ideas o información importantes de lo que lee y escucha, y cierta dificultad para redactar y exponer. En HT se observa una alta disposición hacia el estudio; responsabilidad en su proceso de aprendizaje; ha establecido un hábito de estudio, el cual tiene interés en mejorar. En las dimensiones Mo, PC, RI y RP se muestra que este alumno tiene las mismas características que la alumna del caso anterior (figura 1). Respecto a los procesos grupales se observa que, en cuanto a co, tiene muy baja disposición para comunicarse con sus compañeros; pocas veces procura comunicarse de manera directa y clara; muestra dificultad para escuchar y ser empático. En RI este perfil es similar al anterior, sin embargo, en TE cuenta con mediana disposición, compromiso, interés y disciplina para las tareas grupales; participa con las tareas asignadas y mantiene cierta retroalimentación con sus compañeros de equipo.

Figuras como estas y sus interpretaciones pueden resultar útiles tanto para el alumno como para el profesor y/o tutor, para propiciar entre los estudiantes interacciones de colaboración, respeto, tolerancia, comunicación asertiva, pensamiento crítico y mejora en las actitudes negativas hacia la formación académica y para que los profesores conozcan las actitudes de los estudiantes y con ello proponer acciones psicopedagógicas y comunicacionales para la mejora de las actitudes de los estudiantes ante su formación académica y social, lo que enriquecerá día a día a la comunidad universitaria y su proyección de cara al futuro personal y profesional de los estudiantes.

\section{Conclusiones}

Se cuenta con una versión de instrumento validado y confiable para medir las actitudes hacia el estudio universitario, en el que se distinguen procesos individuales y grupales que permite describir el perfil actitudinal del alumno con tres niveles de clasificación. La validación se realizó mediante el juicio de expertos, descrito en la metodología y los resultados; el análisis de consistencia interna con un coeficiente Alfa de Cronbach mayor a 0.60 en cada dimensión y el total de 0.9660; el análisis factorial exploratorio que permitió identificar procesos individuales y grupales como dos factores. Esta metodología es consistente con Hernández et al. (2006) y con trabajos similares (Bas et al., 2016; García López et al., 2006; Marín-Suárez et al. 2013 y Sáenz et al., 2007).

Aunque se observó una tendencia hacia las respuestas favorables, la información que se obtiene sobre la concepción del deber ser por parte de los estudiantes es por sí misma valiosa; sin embargo, este cuestionario podría mejorar si se revisa la formulación de algunos reactivos y, en su caso, se replantean 
para buscar una respuesta que esté más ligada a la actitud cotidiana y menos a lo que aspira.

Se considera que el instrumento desarrollado será útil para conocer el perfil actitudinal (individual y grupal) de los alumnos tanto al inicio de su formación profesional como en diferentes momentos estratégicos de la misma. Esto le permitirá a la Institución diseñar estrategias encaminadas a mejorar las actitudes de los estudiantes hacia su formación universitaria. Con relación a los cc, conocer el perfil inicial de los alumnos participantes permitirá a los profesores identificar las áreas en las que requieren mayor motivación para tomarlo en cuenta en la planeación de sus clases.

\section{Referencias}

Ajzen, I. (2005). Attitudes, personality and behavior (2a. ed.). Reino Unido: Open University Press McGrawHill Education.

Baron, R. A. y Byrne, D. (2005). Psicología Social (10a. ed.). México: Pearson Prentice Hall.

Bas, G., Kubiato, M. y Murat, A., (2016). Teachers' perceptions towards ICTs in teaching-learning process: Scale Validity and reliability study. Computers in Human Behavior, 61, 176-185.

doi:10.1016/j.chb.2016.03.022

Bohrnstedt, G. W. (2008). Reliability and validity assessment in attitude measurement, en C. Roberts y R. Jowell (Eds.), Attitude Measurement (pp. 143-171). Los Angeles: Sage.

Bosco, M. D. (2009). Nacimiento de un modelo educativo. El Sistema Universidad Abierta y a Distancia de la Universidad Nacional Autónoma de México. Los docentes y su formación (Tesis doctoral). Facultad de Educación: UNED, Madrid.

Fishbein, M. y Ajzen, I. (1975). Belief, attitude, intention and behavior: an introduction to theory and research. Reading, MA: Addison-Wesley.

Fishbein, M. y Ajzen, I. (2010). Predicting and changing behavior: The reasoned action approach. Nueva York: Taylor \& Francis.

García, C. (2013). Estudio de actitudes hacia el aborto en universitarios de Morelos, México. Recuperado de www.lasallep.edu.mx/xihmai/index.php/xihmai/article/down/204/179

García, R. y Hirsch, A. (2006). Construcción de una escala de actitudes sobre ética profesional. Matices, 1(1), 71-82. Recuperado de http://www.aragon.unam.mx/unam/difusion/matices/ejemplares/01.pdf

Gargallo, B., Pérez, C., Fernández, A. y Jiménez, M. A. (2007). La evaluación de las actitudes ante el aprendizaje de los estudiantes universitarios. El cuestionario CEVAPU. Teoría de la Educación. Educación y cultura en la Sociedad de la Información, 8(2), 238-258.

Hernández, R., Fernández-Collado, C. y Baptista, P. (2006). Metodología de la Investigación (4a. ed.), México: Mc GrawHill.

Marin-Suárez, T. y Alarcón, H. (2013). Diseño e implementación de cuestionario para medir las habilidades de aprendizaje colaborativo en estudiantes de ingeniería. XXVI Congreso Chileno de Educación en Ingeniería, en http://sochedi2013.pucv.cl/05 programa.htm

Morales, J. F. (Coord.). (1994). Psicología Social. Madrid: McGraw-Hill/Interamericana de España. 
Peralta, S. C., Ramírez, A. F. y Castaño, H. (2006). Factores resilientes asociados al rendimiento académico en estudiantes pertenecientes a la Universidad de Sucre (Colombia), Psicología desde el Caribe, 17, 196219.

Pliego, O., Contini, L., Odetti, H., Güemes, R. y Tiburzi, M. C. (2004). Las actitudes de los estudiantes universitarios hacia el fenómeno radiactivo, la energía nuclear y sus aplicaciones. Educación Química, 15(2), 142-148.

Rizo, M. (2005). La intersubjetividad como Eje Conceptual para pensar la relación entre comunicación, subjetividad y ciudad. Razón y Palabra, 10(47).

Rodrigues, A. (1976). Psicología Social. México: Trillas.

Sáenz, L., Sánchez-Pérez, L., Luenga, I. y Jarillo, E. (2007). Actitudes de los Estudiantes de Odontología de la UAM-Xochimilco Frente a su Formación Profesional. Archivos Analíticos de Políticas Educativas, 15, 1-16.

Senay, H. (2013). The attitudes of university students towards learning, Procedia - Social and Behavioral Sciences, 83, 947-953. doi:10.1016/j.sbsori.2013.06.177

Topala, I. (2014). Attitudes towards academic learning and learning satisfaction in adult students. Procedia - Social and Behavioral Sciences, 142, 227-234. doi:10.1016/j.sbspro.2014.07.583

UAM-I, CBI. (2008). Sistema Divisional de Estudios a Nivel Licenciatura. Recuperado de http://www.divisional.cbiuami.org/images/consejo/diseno curricular/dis 05.pdf

UAM-I, CBI. (2010-2013). Documentos de seguimiento e informes de Cursos Complementarios. Recuperado de http://ixil.izt.uam.mx/cc/documentos.htm

UAM-I, CBI. (2013). Programa de Cursos Complementarios. Recuperado de http://ixil.izt.uam.mx/cc/programauea.htm 